\title{
Resultados del Taller "Actualización del Plan de Acción para la conservación del huemul Hippocamelus bisulcus (Molina, 1782) en la zona austral de Chile"
}

\author{
Results of Workshop: Updated action plan for the conservation of the \\ andean deer Hippocamelus bisulcus (Molina, 1782) in austral Chile
}

\author{
Alejandro Kusch ${ }^{1}$, Irene Ramírez ${ }^{2}$, Alejandro Vila ${ }^{1}$, Daniela Droguett ${ }^{1}$, Katheryne Paulsen ${ }^{2}$, \\ José Cabello ${ }^{3}$, Fiorella Repetto ${ }^{4}$, Alejandra Silva ${ }^{2} \&$ Michelle Chauveau ${ }^{5}$
}

\section{Resumen}

El Huemul, especie endémica de Chile y la Argentina, es el cérvido más amenazado de la Región Neotropical. El primer Plan Nacional de Conservación de la especie se elaboró el año 2001, dividiendo el trabajo según territorios específicos. Dentro de este marco, en los años 2010 y 2015 se realizaron dos talleres para la elaboración del Plan de la Zona Austral en la Región de Magallanes. Para el último de estos, se realizó una encuesta preliminar para establecer el estado de avance de las acciones propuestas en el 2010 y se aplicó la metodología de Estándares Abiertos para la Práctica de la Conservación para guiar el trabajo. Participaron 32 asistentes de 15 instituciones, que identificaron inconvenientes administrativos, financieros y de recursos humanos para avanzar en la conservación del huemul. Se definió una meta específica para el Plan de la Zona Austral $y$ se priorizaron amenazas en el contexto regional, donde las más relevantes relevantes resultaron ser la competencia con el ganado y la fragmentación de hábitat. Además, se esbozaron indicadores y los principales responsables para facilitar el monitoreo de la gestión del Plan de Acción.

\section{Palabras clave:}

Huemul, estándares abiertos, amenazas, planificación para la conservación

\section{Abstract}

The Huemul, a species endemic to Chile and
Argentina, is the most threatened cervid in the Neotropical Region. The first National Plan for the conservation of the species was developed in 2001 and divided the territory in different working zones. Within this framework, in 2010 and 2015 two workshops for the development of the Plan for Austral Zone Plan took place in the Region of Magallanes. For the last of them, a preliminary survey was conducted to establish the progress of the actions proposed in 2010 and the Open Standards for the Practice of Conservation was applied as a methodology for guiding the work. . Thirty-two attendants from 15 institutions identified administrative, financial and human resources drawbacks to advance in Huemul conservation. A specific goal for the Austral Zone Plan was defined and threats were prioritized according to the regional context, where the most important threats identified were competition with livestock and habitat fragmentation. In addition, indicators and the main responsible for facilitating the

Wildlife Conservation Society-Chile. Balmaceda 586

Punta Arenas. $\bowtie$ akusch@wcs.org

2 Corporación Nacional Forestal. Av. Bulnes 0299, Punta Arenas.

3 Patagoniawildlife, Parcela 71 Villa Andrea, Punta Arenas.

4 Centro Regional Fundación CEQUA, 21 de Mayo 1690, Punta Arenas.

5 Servicio de Evaluación Ambiental, Lautaro Navarro 363, Punta Arenas. 
monitoring of the management of the Action Plan were outlined.

\section{Key Words:}

Huemul, open standards, threats, conservation planning

\section{INTRODUCCIÓN}

El huemul (Hippocamelus bisulcus Molina, 1782) es una especie endémica de Chile y Argentina cuya población y rango de distribución se han reducido considerablemente. La población actual no superaría los 2.500 individuos entre ambos países (Jiménez et al. 2008), distribuyéndose en pequeñas subpoblaciones fragmentadas (Vila et al. 2006). La ocupación, modificación y fragmentación de su hábitat, junto con la caza y la introducción de la ganadería y especies exóticas, han sido mencionadas históricamente como las principales causas que motivaron su declinación (Drouilly 1983, Povilitis 1998, Aldridge \& Montecinos 1998, Smith-Flueck 2000, Serret 2001). En este contexto, el huemul es considerado como el ciervo más amenazado del Neotrópico y fue categorizado como "En Peligro de Extinción" a nivel internacional por la UICN (Jiménez et al. 2008). Además, ha sido incluido en el Apéndice I de las convenciones CITES y CMSBonn. En Chile, se encuentra protegido por la Ley de Caza N 19.473 desde 1929 y en junio del 2006 fue declarado Monumento Natural por el Ministerio de Agricultura. Se encuentra clasificado "En Peligro de Extinción" a nivel nacional ${ }^{1}$.

Considerando la crítica situación que enfrenta la especie, en el año 2001 se publicó el primer Plan Nacional para la Conservación del Huemul en Chile (CONAF \& CODEFF 2001), que fue actualizado posteriormente en el 2007 (CONAF et al. 2009). Dicho Plan propone la conformación de grupos zonales de trabajo para su implementación, en función de las particularidades del rango de distribución de la especie en cada macrozona. En particular, el Grupo de la Zona Austral, correspondiente a la Región de Magallanes, trabajó en un proceso participativo para desarrollar los lineamientos iniciales de un Plan de Acción Zonal en el 2010 (Silva et al. 2011). En paralelo, durante los años 2014 y 2015, la Corporación Nacional
Forestal (CONAF) generó un Plan de Acción de mediano plazo (2015-2018) para la conservación de la especie, para guiar el accionar interno de la institución, teniendo como marco el Plan Nacional. En este contexto, los días 21 y 22 de octubre de 2015 se organizó, en la ciudad de Punta Arenas, el Taller "Actualización del Plan de Acción para la Conservación del huemul en la Zona Austral". Las organizaciones convocantes de esta iniciativa, CONAF y Wildlife Conservation Society Chile (WCS), propusieron revisar, actualizar y refinar la propuesta de Plan de Acción Zonal elaborada en el 2010.

En el presente documento sintetizamos las recomendaciones y conclusiones obtenidas durante el taller, como un insumo para avanzar en desarrollar un Plan de Acción coherente, alineado con los Planes de mayor escala vigentes y consensuado entre todas las partes interesadas.

\section{METODOLOGÍA}

\section{Encuesta a los asistentes}

Con antelación a la realización del taller, se envió a los invitados una encuesta para determinar si: i) habían participado del taller anterior, ii) tenían conocimiento sobre las Actas del mismo, iii) si identificaban nuevas amenazas para la especie y iv) habían desarrollaron acciones concretas en la Zona Austral desde 2010 a la fecha. De esta forma, se esperaba compilar y evaluar los avances de las propuestas de acción desarrolladas en el taller previo.

Desarrollo del taller: Expectativas, meta y objetivos, y priorización de acciones por amenaza

El enfoque metodológico del taller estuvo basado en los Estándares Abiertos para la Práctica de la Conservación (CMP 2013) y en dinámicas de trabajo participativas. Se contó con la presencia de 32 participantes (67\% de los invitados) que representaron a 15 instituciones, y un investigador independiente. De los 32 asistentes, 24 (75\%) participaron en las actividades de trabajo grupal del taller.

La primera actividad realizada en el taller 
fue la presentación de los participantes y sus expectativas. La información obtenida en este ejercicio sirvió como insumo para comprender las propuestas de cada institución y sus conocimientos sobre las temáticas a tratar. Las palabras claves mencionadas por cada persona en relación a su expectativa fueron ordenadas y evaluadas según su frecuencia de mención.

\section{Definición de una meta y objetivos}

Con el fin de elaborar el enunciado de una meta de conservación específica para el Plan de Acción del Huemul en la Zona Austral, se le instruyó a la audiencia que propusieran palabras claves que deberían estar presentes en el enunciado de la meta de conservación, y con las cuales se pueda redactar dicha meta para la conservación del huemul.

Posteriormente, se realizó un ejercicio grupal para proponer acciones en el marco de tres ejes para estructurar el Plan de Acción. Los participantes se dividieron en tres grupos: a) políticas públicas y legislación; b) educación y comunicaciones; y c) investigación, monitoreo y manejo. Cada grupo revisó la lista y orden de prioridad de amenazas directas que se identificaron en el taller del año 2010, para definir si era necesario realizar cambios. A partir del ranking de prioridad obtenido, el trabajo de cada grupo se concentró en las tres amenazas más prioritarias, para proponer, al menos, tres actividades orientadas a abordarlas. Como parte de las instrucciones a los asistentes, se puso énfasis en que las propuestas fueran factibles tanto política, como social- y organizacionalmente (capacidad institucional, recursos humanos y económicos disponibles). Finalmente, se solicitó que los grupos identificaran las instituciones $\mathrm{u}$ organizaciones que debieran estar a cargo de realizar y coordinar dichas actividades.

\section{RESULTADOS}

\section{Encuesta a los asistentes}

Del total de encuestas enviadas previamente a los potenciales asistentes al taller $(n=48)$, sólo hubo respuesta de ocho personas $(16,7 \%)$, tres del ámbito público y cinco del privado. Cinco de ellas habían participado del taller del año 2010 y tenían conocimiento de las actas. Sólo una de las instituciones encuestadas (CONAF) identificó una amenaza nueva en relación a las planteadas en el

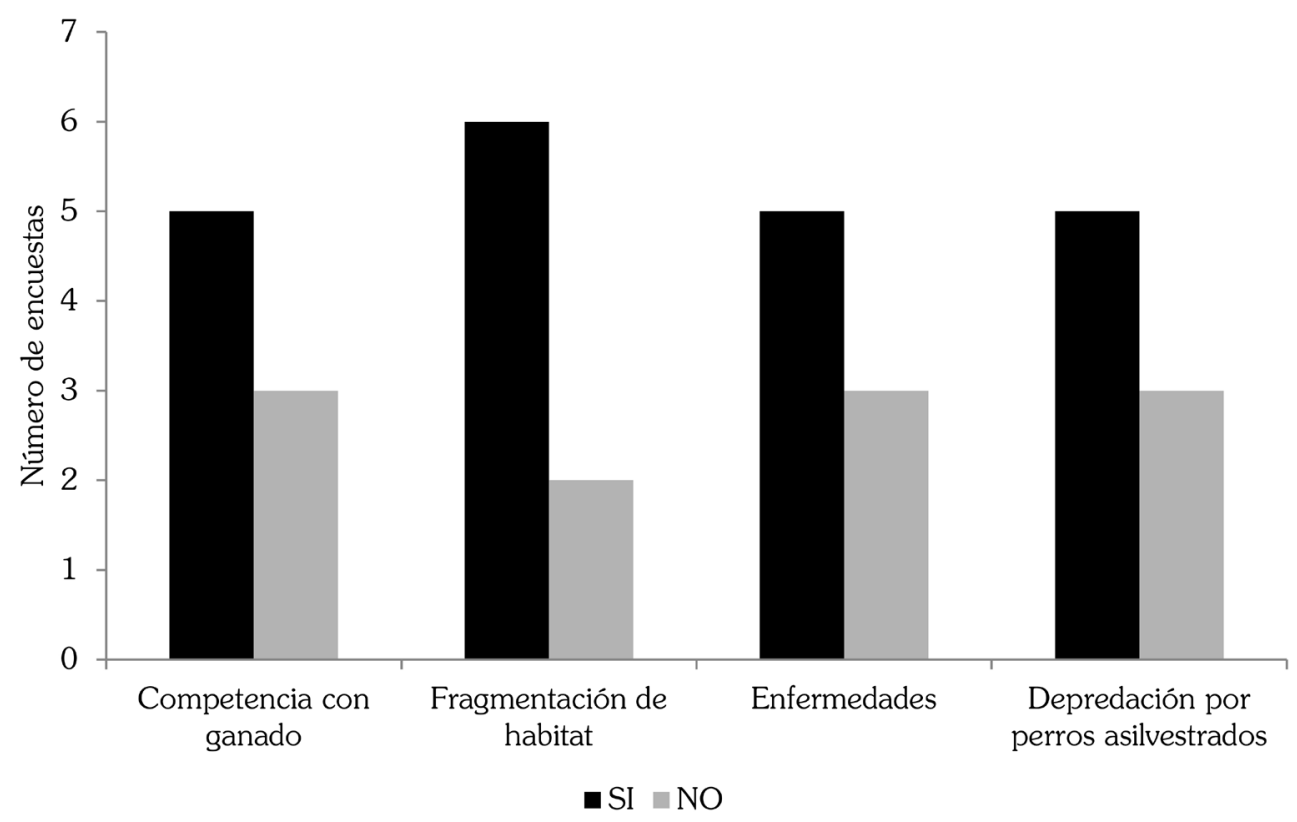

Fig. 1. Acciones implementadas por los encuestados, en los últimos 5 años, en vinculación con la mitigación de las cuatro amenazas prioritarias que fueron identificadas en el 2010. 


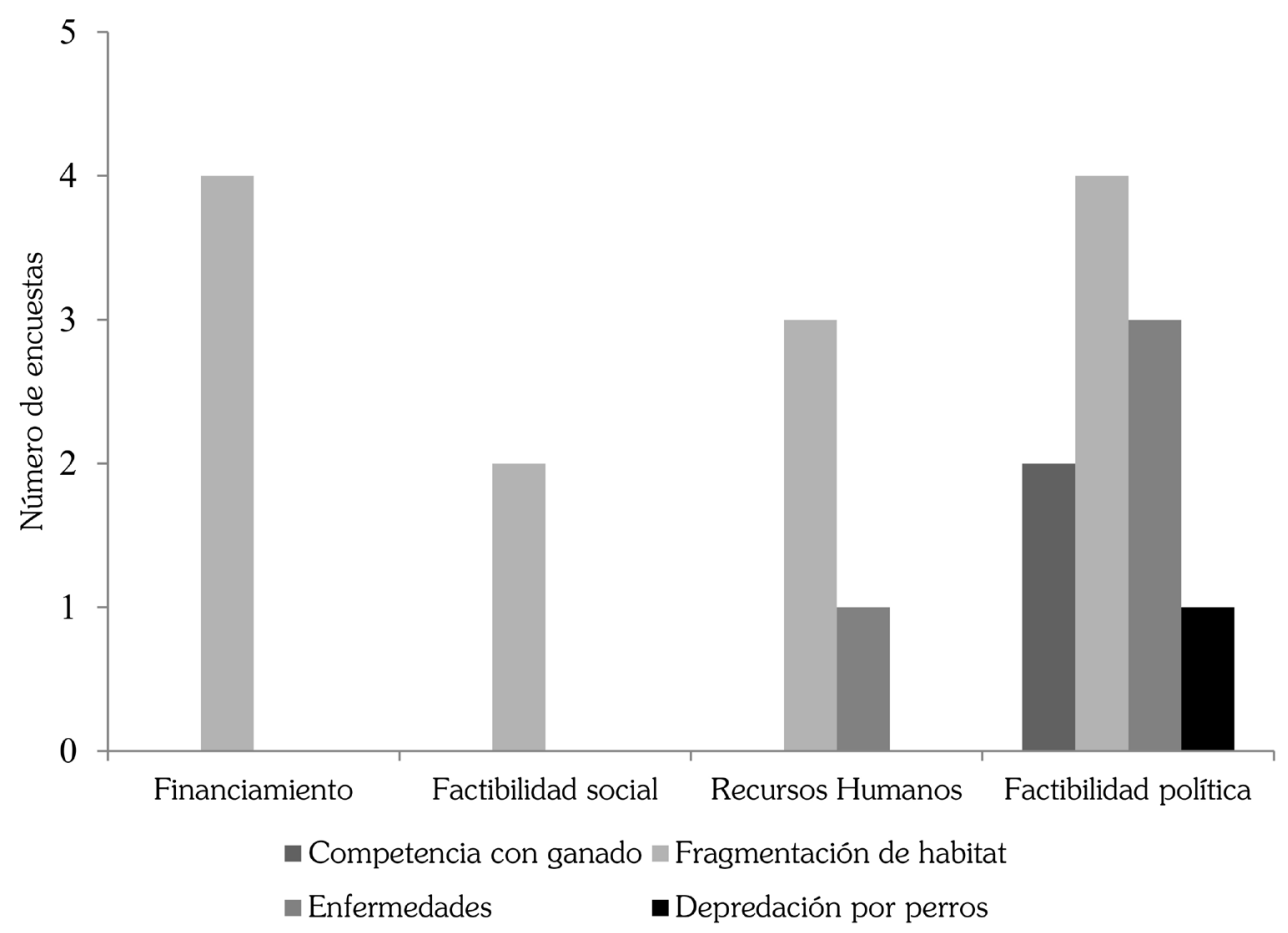

Fig. 2. Limitantes para desarrollar acciones que fueron mencionadas por los encuestados en función de las cuatro amenazas principales identificadas para el huemul en el 2010.

2010. En general, los encuestados reconocieron haber implementado acciones de conservación para mitigar las cuatro principales amenazas planteadas en el 2010 (Fig. 1). La nueva amenaza propuesta fue el hostigamiento de huemules por parte de personas/turistas, acción que ha sido observada en áreas silvestres protegidas.

A través de las encuestas, además se identificó cuáles fueron las principales limitantes para avanzar en la implementación de acciones para contrarrestar las amenazas. Estas se relacionaron con la factibilidad político-administrativa $(37,5 \%$ de las respuestas), falta de profesionales/recursos humanos, déficit en el financiamiento (50\%), y contexto social inadecuado $(12,5 \%$ de las respuestas). Al especificar la relación entre estas limitantes y las amenazas específicas (ver Silva et al. 2011, Fig. 2), los encuestados manifestaron que para enfrentar la pérdida o fragmentación de hábitat del huemul, todas las limitantes dificultaron el desarrollo de acciones. Por su parte, la factibilidad político-administrativa fue identificada como una causa que incide sobre la ejecución de acciones para mitigar todas las amenazas, y afectó principalmente a la implementación de acciones para contrarrestar la competencia con ganado y la depredación por perros, mientras que la limitante de recursos humanos fue mencionada en relación a la amenaza de enfermedades que atacan a los huemules. Adicionalmente, los encuestados mencionaron otras limitantes adicionales, entre las que se destacan: falta de desarrollo institucional, falta o debilidad normativa, baja consistencia entre los aspectos técnicos y políticos para la toma de decisiones, priorización de otras especies en distintos Ministerios, baja coordinación interna o intrasectorial y baja coordinación intersectorial.

\section{Expectativas del taller}

Una vez iniciado el taller, 23 participantes manifestaron sus expectativas de trabajo y se identificaron nueve grupos de expectativas concretas a partir de las opiniones compartidas en las presentaciones (Fig. 3). Entre ellas, se destaca el interés en conocer información general y actualizada sobre los huemules en la zona austral (22\%), generar avances sobre el Plan de Acción 
Tabla 1. Priorización de acciones para la disminución de amenazas que operan sobre el huemul desde la perspectiva de los diferentes grupos de trabajo.

\begin{tabular}{lll}
\hline & Grupos de trabajo y acciones priorizadas & \\
\hline \multicolumn{1}{c}{ Políticas Públicas } & \multicolumn{1}{c}{ Educación y Divulgación } & Investigación, Monitoreo y Manejo \\
\hline Fragmentación de hábitat & Competencia con ganado & Competencia con ganado \\
Hostigamiento antrópico & Depredación y acoso por perros & Enfermedades \\
Competencia con ganado & Fragmentación de hábitat & Fragmentación de hábitat \\
\hline
\end{tabular}

anterior $(17 \%)$, proponer indicadores de logro para cada actividad por amenaza (13\%) y generar acciones específicas para territorios concretos (13\%).

\section{Definición de una meta y objetivos}

Los participantes propusieron las siguientes palabras clave: huemul, zona austral, aumentar la población, conservar, amenazas, estado de conservación, distribución, recuperar, abundancia, 2015-2020, poblaciones funcionales, restaurar y poblaciones saludables. En base a ellas, y, tomando como punto de partida las recomendaciones del taller anterior (Silva et al. 2011), se decidió trabajar sobre una meta enfocada específicamente en la realidad de la Zona Austral, ya que previamente se había tomado como base la meta general del Plan Nacional: "Revertir la declinación poblacional del huemul en Chile".

Respecto a la declinación poblacional en la Zona Austral, la audiencia convino en que si bien han existido eventos específicos que pudieron haber provocado mortalidad local de individuos, como el desprendimiento de una gran masa de hielo que produjo una inundación en el área ocupada por huemules en el fiordo Bernardo el año 2010 (M. Ruiz com. pers.), o la mortalidad asociada a lesiones podales en el fiordo Témpanos ( $48^{\circ} 41^{\prime} \mathrm{S}, 73^{\circ} 59^{\prime} \mathrm{W}$ ) entre 2005 y 2010 (A. Silva com. pers), actualmente, no se dispone de datos cuantitativos que permitan confirmar la declinación poblacional a nivel zonal. Por otro lado, también se mencionó que el control local del ganado asilvestrado pudo contribuir a la reocupación de áreas previamente utilizadas por huemules dentro de los Parques Nacionales Torres del Paine y Bernardo O־Higgins. Asimismo, para el caso de Torres del Paine, el incremento en la observación de crías registrado en los últimos años y el aumento de avistamientos directos de ejemplares en distintos sectores del parque, puede ser un indicio de una potencial recuperación poblacional (G. Garay com. pers). Finalmente, los pocos antecedentes de distribución y abundancia disponibles para la especie indican que las poblaciones más abundantes y mejor conservadas se encuentran en la zona austral y principalmente dentro de áreas protegidas (Vila et al. 2006).

En función de los antecedentes anteriores, según el criterio de los expertos presentes, se acordó que la situación en la región no se corresponde completamente con la meta planteada en el Plan Nacional, ya que no existe una declinación poblacional aparente en la zona austral. Sin embargo, se manifestó la necesidad de mejorar el estado de conocimiento de la distribución y abundancia de la especie. A partir de esta discusión, un subgrupo elaboró el siguiente enunciado de meta que fue posteriormente validado por los demás participantes: "Las poblaciones de huemules en la zona austral, durante el periodo 2016-2020, al menos, se conservan saludables y funcionales, a través del mejoramiento del conocimiento de la distribución del huemul, y de las contribuciones significativas para la mitigación de amenazas".

\section{Priorización de acciones por amenaza}

Los tres grupos de trabajo priorizaron las amenazas sobre las poblaciones de huemules (Tabla 1) y para cada una de ellas se determinaron acciones concretas. Entre las nueve amenazas priorizadas, la que fue identificada como más prioritaria fue la competencia con ganado. En segundo lugar se identificó la fragmentación de hábitat $\mathrm{y}$, finalmente, en tercer lugar se propuso el hostigamiento antrópico, la depredación 
por perros y las enfermedades, ya que las tres obtuvieron la misma valoración en la priorización.

Grupo 1: Políticas públicas

A. Acciones propuestas para disminuir la pérdida y fragmentación de hábitat

1. Modificación del literal p) del Art. N³, del DS 40 de 2012, Reglamento del Sistema de Evaluación de Impacto Ambiental. Este indica que aquellos proyectos o actividades que son susceptibles de causar impacto ambiental deben someterse al Sistema de Evaluación de Impacto Ambiental (SEIA), mientras que la letra p) se refiere a la "ejecución de obras, programas o actividades en parques nacionales, reservas nacionales, monumentos naturales, reservas de zonas vírgenes, santuarios de la naturaleza, parques marinos, reservas marinas o en cualesquiera otras áreas colocadas bajo protección oficial, en los casos en que la legislación respectiva o permita". Dentro de estas áreas se podría incorporar el concepto de "áreas con presencia de especies En Peligro", lo cual generaría la obligatoriedad de ingreso al SEIA de proyectos a desarrollar en áreas que, aun cuando no estén bajo protección oficial, sean reconocidas por albergar una población importante de especies amenazadas.

2. Revisar y proponer modificaciones a la normativa para la conservación de hábitat en áreas productivas silvoagropecuarias (norma e instrumentos de incentivo similares al Sistema de Incentivos para la Sustentabilidad Agroambiental de los Suelos Agropecuarios, para ser aplicados a la vida silvestre).

3. Promover al huemul como un ítem específico en la Política Regional de Biodiversidad.

4. Modificación de los planes de manejo de las áreas silvestres protegidas con presencia de la especie, en particular la zonificación, para mejorar las restricciones de uso en zonas con presencia de huemul.
B. Acciones propuestas para disminuir el hostigamiento antrópico

1. Promoción de buenas prácticas para distintas actividades antrópicas, como recreación y obras públicas. En particular se promoverá el trabajo interdisciplinario entre expertos locales y la Dirección de Vialidad (Ministerio de Obras Públicas), junto con la capacitación de consultores externos, con el objetivo de ejecutar medidas que aseguren el movimiento de individuos entre poblaciones, ante la construcción de caminos en áreas con presencia de huemules.

2. Implementación de reglas indicativas o normativas en la zonificación de ASP.

C. Acciones propuestas para disminuir la competencia con ganado

1. Erradicación de ganado doméstico y/o asilvestrado de las ASP.

2. Erradicación de ganado asilvestrado en las zonas que están fuera de las ASP y que tienen presencia de huemul.

3. Promoción de buenas prácticas de manejo predial (agroforestal) en áreas con presencia de huemul. En particular, promover un manual de buenas prácticas para el manejo predial en zonas con huemules, como por ejemplo en Isla Riesco y la Península de Brunswick.

4. Solicitar la revisión de la eficacia de las denuncias efectuadas a los juzgados de policía local por la rotura de cercos ganaderos y otros factores que comprometan la conservación del huemul.

Grupo 2: Educación y divulgación

A. Acciones propuestas para disminuir la competencia con ganado

1. Difundir buenas prácticas de manejo ganadero en áreas con huemules.

2. Sensibilizar a las autoridades para mantener el financiamiento de acciones de erradicación de ganado.

3. Elaborar material de difusión relacionado 
al tema.

B. Acciones propuestas para disminuir la depredación y acoso por perros

1. Fomentar la participación ciudadana, en particular a las poblaciones humanas cercanas a huemules.

C. Acciones propuestas para disminuir la fragmentación de hábitat

1. Fomentar buenas prácticas en relación a la construcción de caminos y cercos (cercos amigables con la fauna silvestre).

2. Capacitación a técnicos de los servicios públicos que integran los comités técnicos del Servicio de Evaluación de Impacto Ambiental (SEIA).

3. Diseño e instalación de señalética apropiada en caminos que cruzan áreas con presencia de huemules.

Grupo 3. Investigación, monitoreo y manejo

A. Acciones propuestas para disminuir la competencia con ganado

1. Elaborar un Plan de Erradicación de ganado asilvestrado en ASP.

2. Determinar la distribución y abundancia de ganado en áreas con presencia de huemul y desarrollar una plataforma geomática como herramienta de apoyo al Plan de Erradicación referido en el punto anterior.

3. Monitoreo de las acciones de erradicación.

4. Evaluación de interacción ecológica con ganado doméstico.

B. Acciones propuestas para disminuir las enfermedades

1. Establecimiento de un protocolo de monitoreo sanitario de las poblaciones de huemules en la Región de Magallanes.

2. Monitoreo sanitario y evaluación de los factores de riesgo de enfermedades potenciales del huemul.

3. Evaluación sanitaria de ganado doméstico en áreas de influencia ecológica de huemul.
4. Desarrollo de un protocolo de acción ante eventos de mortalidad de huemul.

C. Acciones propuestas para disminuir la fragmentación de hábitat

1. Estandarizar un plan de monitoreo para la Región de Magallanes y difundir a la comunidad y los actores claves.

2. Monitoreo de las poblaciones de huemul en áreas potenciales de distribución, priorizando áreas con proyección de proyectos que puedan generar fragmentación.

3. Exigir planes de monitoreo de huemul previo a la ejecución de proyectos (SEIA).

4. Evaluar el impacto de la construcción de caminos.

5. Generar medidas para reducir impacto de caminos.

6. Incrementar medidas de restauración en hábitat del huemul y estandarizar el monitoreo post-incendios.

7. Estudio genético para conocer la variabilidad genética de huemul, teniendo como prioridad el área de Cabo Froward e Isla Riesco.

Indicadores de gestión de amenazas

Se propusieron 35 indicadores para llevar a cabo el seguimiento y logro de cada una de las acciones propuestas en relación a cada amenaza (Anexo 1). Además se seleccionaron las instituciones responsables del cumplimiento de cada acción.

\section{DISCUSIÓN Y CONCLUSIONES}

Las expectativas antes del trabajo propio del taller mostraron que una fracción importante de los asistentes asistió con la intención de informarse sobre aspectos diversos del huemul en Magallanes (Fig. 3). En un público de asistentes que puede considerarse profesionales del área de la biología de conservación, investigadores y/o manejadores de recursos naturales, la necesidad de información indica que entre pares o instituciones no existe suficiente divulgación de las actividades que se llevan a cabo para la conservación del huemul. 
Relevar la importancia cultural de la especie

Participación más integradora

Actualización de la información sobre proyectos

Establecimiento de responsables de acciones

Identificación de metas especificas

Acciones específicas por tipo territorio

Propuesta de indicadores

Avances en un Plan de Conservación

Información general
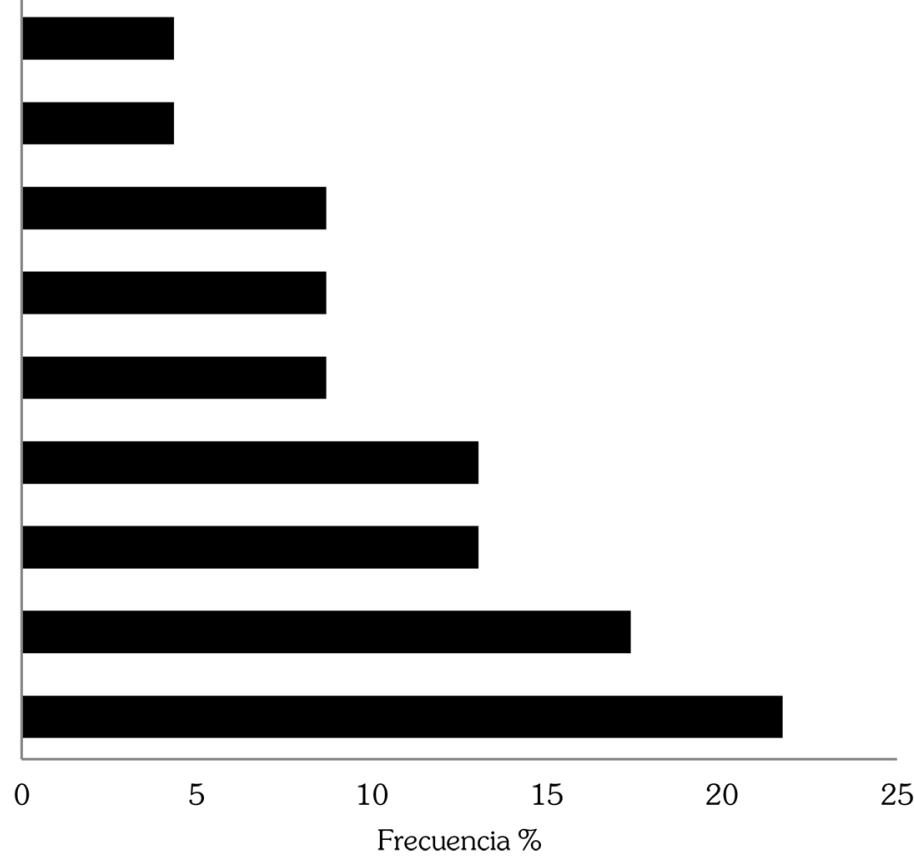

Fig. 3. Frecuencia de las expectativas planteadas por los participantes en el encuentro.

Los grupos presentaron sus resultados en plenario y se realizó una sesión de discusión respecto a los temas planteados. Se resaltaron algunas temáticas como la necesidad de evaluar las metodologías para el monitoreo de las poblaciones de huemul, poniendo énfasis en la coordinación entre CONAF y el SAG para la autorización de permisos de investigación. Sobre el mismo punto, se sugirió que para avanzar en la divulgación de los resultados de investigación y monitoreo, se solicite a los investigadores que difundan sus resultados a la comunidad, más allá de la preparación de manuscritos técnicos o publicaciones científicas. Con el objeto de priorizar y trabajar en líneas de investigación en relación al huemul y otras acciones priorizadas en el marco del Plan de Acción Zonal Austral, se propuso crear un comité de expertos.

En aspectos de divulgación y educación, se resaltó la necesidad de utilizar la red de comités regionales que reúnen a los actores vinculados a temáticas de conservación de la biodiversidad, por lo que se sugirió la utilización del Comité Regional de Educación Ambiental (CREA), como un medio para relevar las actividades y acciones de divulgación y educación del Plan Zonal.

Finalmente, el trabajo desarrollado en el taller para la conservación del huemul en la zona austral de Chile, reflejó que las particularidades de la región permiten condiciones favorables para las poblaciones de huemul en comparación con su distribución nacional y que esto debe estar reflejado en las metas del plan de acción a nivel local, y de esta forma lograr coherencia con las acciones. La evaluación de las medidas sugeridas en el taller anterior indican que si bien todos los integrantes reconocen haber implementado parte de las acciones comprometidas, aún existen importantes necesidades y vacíos de información relacionados con las políticas públicas, educación ambiental e investigación científica. Más aún, si se comparan las acciones realizadas al interior y fuera de las áreas protegidas, se discutió entre los asistentes que la conservación de esta especie se aprecia más frágil cuando se reconocen limitantes administrativas, ya que no siempre se ejecutan acciones coordinadas, o bien se hacen necesarios presupuestos que no son identificados como prioritarios por las instituciones encargadas. Sin embargo, existe al menos un ejemplo de cooperación público-privado que dio señales positivas en un trabajo de conservación a mediano plazo donde se pudo determinar una tendencia positiva de la población de huemules en relación a la erradicación de ganado (ver Briceño et al. 2013). 
En particular, algunas de las acciones acordadas para minimizar las amenazas sobre el huemul son específicas para la especie y tienen relación a espectos de sanidad animal y monitoreo de las poblaciones. Pero también, se identificaron otras medidas que son orientadas a la conservación del huemul pero pueden servir para salvaguardo de otras especies. Algunas de estas medidas tienen relación al control de fauna asilvestrada, manejo de áreas de uso publico para evitar incendios, disturbios a animales y señalética en general.

El trabajo futuro para la conservación del huemul en la zona austral de Chile no sólo debe considerar los aspectos acordados en el taller. En el mismo, se alcanzó a discutir sobre el alcance geográfico de las principales amenazas pero no se pudo asociar en una cartografía las acciones para repeler amenazas. También se discutió sobre la viabilidad económica y la gestión en polítias públicas regionales. La conformación del grupo de expertos $e$ instituciones vinculadas al huemul debe ser una prioridad ya que de esta forma se dará impulso a la ejecución coordinada de la amplia gama de medidas presentadas, y de la gestión de recursos.

\section{AGRADECIMIENTOS}

Los autores agradecen la participación de todos los representantes de las instituciones que asistieron al taller y en particular a Claudia Silva por la rigurosa revisión del manuscrito. También a dos revisores anónimos que aportaron valiosos comentarios al manuscrito.

\section{LITERATURA CITADA}

Aldridge, D., \& Montecinos, L. (1998). Avances en la conservación del huemul en Chile. En V. Valverde (Ed.), La conservación de la Fauna Nativa en Chile. Logros y perspectivas (pp. 133-148) CONAF, Ministerio de Agricultura, Santiago.

Briceño, C., Knapp, L. A., Silva, A., Paredes, J., Avendaño, I., Vargas, A., Sotomayor, J., \& Vila, R. A. (2013). Detecting an increase in an Endangered huemul Hippocamelus bisulcus population following removal of cattle and cessation of poaching. Oryx, 47 (2), 273-279.
CMP (Conservation Measures Partnership) (2009). Estándares Abiertos para la práctica de la conservación. Versión 2.0. 2007. African Wildlife Foundation, The Nature Conservancy, Wildlife Conservation Society, World Wildlife Fund.

CONAF \& CODEFF (2001). Plan para la Conservación del Huemul del Sur, Hippocamelus bisulcus, en Chile.

CONAF, SAG, CONAMA (2009). Plan Nacional de Conservación del huemul (Hippocamelus bisulcus, Molina 1782) en Chile. 20082012. Taller Participativo para la elaboración del Plan, Agosto 2007.

Drouilly, P. (1983). Recopilación de antecedentes biológicos y ecológicos del huemul chileno y consideraciones sobre su manejo. Boletín Técnico 5, CONAF, Santiago.

Jiménez, J., Guineo, O., Corti, P., Smith, J. A., Flueck, W., Vila, A., Gizejewski, Z.,...Geist, V. (2008). Hippocamelus bisulcus. En IUCN 2010. IUCN Red List of Threatened Species. Version 2010.4 URL: http//www. iucnredlist.org

Povilitis, A. (1998). Characteristics and conservation of a fragmented population of huemul Hippocamelus bisulcus in central Chile. Biological Conservation, 86, 97104.

Silva, C., (2011). Actas De Taller: hacia un Plan para la Conservación del huemul Hippocamelus Bisulcus, (Molina, 1782) En la Zona Austral de Chile. (11-12 De Noviembre 2010, Punta Arenas). Anales Instituto Patagonia, 39(1), 119-136.

Serret, A. (2001). El huemul fantasma de la Patagonia. Zagier y Urruty Publications.

Smith-Flueck, J. A. (2000). La situación actual del huemul Patagónico. En N. I. Díaz \& J. A. Smith.Flueck (Eds.), El Huemul Patagónico, un misterioso cérvido al borde de la extinción (pp. 67-147). Buenos Aires: Literature of Latin América, Buenos Aires.

Vila, A. R, López, R., Pastore, H., Faúndez, R., \& Serret, A. (2006). Current distribution and conservation of the huemul (Hippocamelus bisulcus) in Argentina and Chile. Mastozoología Neotropical, 13(2), 263-269. 
ANEXO 1. Indicadores de cumplimiento y responsabilidades identificadas para cada amenaza según el grupo de trabajo.

\section{A. Políticas públicas}

\begin{tabular}{|c|c|c|c|c|}
\hline Amenaza & Acción & Responsables & Actividades & Indicadores \\
\hline \multirow[t]{4}{*}{ Perdida hábitat } & $\begin{array}{l}\text { Modificación del literal } \\
\text { p) del Art. Nº } 3 \text {, del } \\
\text { DS } 40 \text { de } 2012 \text {, } \\
\text { Reglamento del Sistema } \\
\text { de Evaluación de } \\
\text { Impacto Ambiental }\end{array}$ & SEA & Elaboración de propuesta & $\begin{array}{l}\text { Propuesta y seguimiento por el } \\
\text { SEA regional } \\
\text { Revisión/reporte técnico de los } \\
\text { documentos (estrategia y plan } \\
\text { de acción) existentes sobre el } \\
\text { tema huemul }\end{array}$ \\
\hline & $\begin{array}{l}\text { Normativa tipo SIRSD } \\
\text { vida silvestre }\end{array}$ & SAG & $\begin{array}{l}\text { Trabajo de gabinete y } \\
\text { reuniones de trabajo con } \\
\text { CORE y Seremis }\end{array}$ & $\begin{array}{l}\text { Propuesta y seguimiento } \\
\text { desde XII región a nivel central } \\
\text { (DIPROREN) }\end{array}$ \\
\hline & $\begin{array}{l}\text { Implementar Política } \\
\text { Regional Biodiversidad }\end{array}$ & MMA & Coordinación interna & $\begin{array}{l}\text { Política RB implementada con } \\
\text { tema huemul }\end{array}$ \\
\hline & $\begin{array}{l}\text { Modificación planes de } \\
\text { manejo y zonificación }\end{array}$ & CONAF & $\begin{array}{l}\text { Elaboración TTR para } \\
\text { elaboración de planes de } \\
\text { manejo en aquellos casos } \\
\text { donde no existen }\end{array}$ & $\begin{array}{l}\text { Propuesta de modificación de } \\
\text { zonificaciones en ASP con } \\
\text { huemules }\end{array}$ \\
\hline \multirow[t]{2}{*}{ Hostigamiento } & Buenas prácticas MOP & MMA, CONAF & $\begin{array}{l}\text { Reuniones/coordinación } \\
\text { Implementación de Medidas }\end{array}$ & Reuniones inter sectoriales \\
\hline & & & del MMA & $\begin{array}{l}\text { Medidas propuestas } \\
\text { implementadas }\end{array}$ \\
\hline \multirow[t]{6}{*}{$\begin{array}{l}\text { Competencia } \\
\text { con ganado }\end{array}$} & Erradicación en ASP & CONAF & $\begin{array}{l}\text { Generar Plan de } \\
\text { Erradicación }\end{array}$ & $\begin{array}{l}\text { Plan preparado a un año } \\
\text { con calendarización de } \\
\text { extracción por ASP y mapa de } \\
\text { distribución de ganado } \\
\% \text { de ganado erradicado por } \\
\text { ASP (a } 5 \text { años) }\end{array}$ \\
\hline & & & $\begin{array}{l}\text { Implementar el Plan } \\
\text { para Recuperar hábitat }\end{array}$ & $\begin{array}{l}\text { \% cumplimiento del Plan } \\
\text { Ocupación de hábitat por } \\
\text { huemul (10 años) }\end{array}$ \\
\hline & $\begin{array}{l}\text { Erradicación fuera ASP } \\
\text { (predios privados) }\end{array}$ & $\begin{array}{l}\text { Productores y } \\
\text { SAG }\end{array}$ & $\begin{array}{l}\text { Gestión de acciones de } \\
\text { manera intrasectorial }\end{array}$ & $\begin{array}{l}\% \text { de ganado erradicado (a } 5 \\
\text { años) }\end{array}$ \\
\hline & $\begin{array}{l}\text { Erradicación fuera ASP } \\
\text { (predios fiscales) }\end{array}$ & BBNN & $\begin{array}{l}\text { Gestión de acciones de } \\
\text { manera intrasectorial }\end{array}$ & $\begin{array}{l}\text { \% de ganado erradicado (a } 5 \\
\text { años) }\end{array}$ \\
\hline & $\begin{array}{l}\text { Incentivo a las Buenas } \\
\text { práctica }\end{array}$ & $\begin{array}{l}\text { Seremi } \\
\text { Agricultura }\end{array}$ & $\begin{array}{l}\text { Diseño o maqueta de un } \\
\text { Manual }\end{array}$ & $\begin{array}{l}\text { Propuesta de manual o buenas } \\
\text { prácticas ganaderas }\end{array}$ \\
\hline & Fomentar las denuncias & Superintendencia & $\begin{array}{l}\text { Elaboración de una } \\
\text { estrategia para denunciar } \\
\text { ilícitos y el seguimiento }\end{array}$ & Número de denuncias \\
\hline
\end{tabular}




\section{B. Educación y Divulgación}

\begin{tabular}{|c|c|c|c|c|}
\hline Amenaza & Acción & Responsables & Actividades & Indicadores \\
\hline \multirow[t]{3}{*}{$\begin{array}{l}\text { Competencia con } \\
\text { ganado }\end{array}$} & $\begin{array}{l}\text { Difundir buenas } \\
\text { prácticas de manejo } \\
\text { ganadero }\end{array}$ & SAG - CONAF & $\begin{array}{l}\text { Elaboración de un Manual } \\
\text { Lanzamiento del Manual } \\
\text { con los actores involucrados }\end{array}$ & $\begin{array}{l}\text { Propuesta de manual o buenas } \\
\text { prácticas }\end{array}$ \\
\hline & $\begin{array}{l}\text { Sensibilizar a } \\
\text { las autoridades } \\
\text { para mantener el } \\
\text { financiamiento }\end{array}$ & $\begin{array}{l}\text { SAG } \\
\text { CONAF } \\
\text { MMA }\end{array}$ & Reunión de gestión & Actas de reuniones \\
\hline & $\begin{array}{l}\text { Elaborar material de } \\
\text { difusión }\end{array}$ & $\begin{array}{l}\text { CONAF - WCS } \\
\text { - AMA Torres } \\
\text { del Paine }\end{array}$ & Reunión de Coordinación & Propuesta de Acción \\
\hline \multirow[t]{2}{*}{$\begin{array}{l}\text { Depredación y } \\
\text { acoso por perros }\end{array}$} & $\begin{array}{l}\text { Elaboración de un } \\
\text { Manual de Tenencia } \\
\text { Responsable }\end{array}$ & $\begin{array}{l}\text { Municipios } \\
\text { SAG }\end{array}$ & Elaboración del Manual & $\begin{array}{l}\text { Manual de tenencia } \\
\text { responsable }\end{array}$ \\
\hline & $\begin{array}{l}\text { Fomentar la } \\
\text { participación ciudadana }\end{array}$ & $\begin{array}{l}\text { Municipios } \\
\text { SAG }\end{array}$ & Taller de difusión & Acta del Taller \\
\hline \multirow[t]{3}{*}{$\begin{array}{c}\text { Fragmentación de } \\
\text { hábitat }\end{array}$} & $\begin{array}{l}\text { Fomentar buenas } \\
\text { prácticas en relación a } \\
\text { caminos y cercos }\end{array}$ & $\begin{array}{l}\text { SAG CONAF } \\
\text { MMA }\end{array}$ & $\begin{array}{l}\text { Taller de trabajo } \\
\text { intersectorial para la } \\
\text { elaboración del Manual de } \\
\text { buenas practicas } \\
\text { Difusión del Manual }\end{array}$ & $\begin{array}{l}\text { Convenio entre MOP y } \\
\text { servicios públicos } \\
\text { Número de atropellos } \\
\text { Proyectos que consideran } \\
\text { buenas prácticas } \\
\text { Km. de cercos amigables } \\
\text { construidos } \\
\text { Número de animales } \\
\text { enganchados o con problemas }\end{array}$ \\
\hline & $\begin{array}{l}\text { Capacitación a técnicos } \\
\text { de los servicios públicos } \\
\text { en el SEIA }\end{array}$ & $\begin{array}{l}\text { SAG CONAF } \\
\text { SEA }\end{array}$ & $\begin{array}{l}\text { Capacitar al Comité Técnico } \\
\text { de Evaluación Ambiental }\end{array}$ & $\begin{array}{l}\text { Profesionales capacitados del } \\
\text { SEIA } \\
\text { Profesionales capacitados del } \\
\text { MOP }\end{array}$ \\
\hline & $\begin{array}{l}\text { Diseño e instalación de } \\
\text { señalética }\end{array}$ & MOP CONAF & $\begin{array}{l}\text { Elaborar propuesta de } \\
\text { señalética }\end{array}$ & Señalética Instaladas \\
\hline
\end{tabular}




\section{Investigación, Monitoreo y Manejo}

\begin{tabular}{|c|c|c|c|c|}
\hline Amenaza & Acción & Responsables & Actividades & Indicadores \\
\hline \multirow[t]{5}{*}{ Enfermedades } & $\begin{array}{l}\text { Elaboración de } \\
\text { un protocolo } \\
\text { de muestreo } \\
\text { y análisis de } \\
\text { huemul para el } \\
2016\end{array}$ & $\begin{array}{l}\text { Universidades } \\
\text { ONGs }\end{array}$ & $\begin{array}{l}\text { Reunión de trabajo } \\
\text { Elaboración de protocolo } \\
\text { Elaboración de convenios }\end{array}$ & $\begin{array}{l}\text { Protocolos elaborados } \\
\text { Convenios firmados }\end{array}$ \\
\hline & $\begin{array}{l}\text { Toma de } \\
\text { muestras no } \\
\text { invasivas }\end{array}$ & CONAF & $\begin{array}{l}\text { Implementación de } \\
\text { protocolos }\end{array}$ & $\begin{array}{l}\text { Número de muestras analizadas } \\
\text { por sitio } \\
\text { Poblaciones sanas para el } 2025\end{array}$ \\
\hline & $\begin{array}{l}\text { Toma de } \\
\text { muestras a } \\
\text { poblaciones } \\
\text { de ganado } \\
\text { doméstico }\end{array}$ & SAG & $\begin{array}{l}\text { Identificación de sitios con } \\
\text { presencia de huemules y } \\
\text { ganado } \\
\text { Solicitar a SAG muestras y } \\
\text { análisis de resultados de los } \\
\text { sectores }\end{array}$ & $\begin{array}{l}\text { Diagnóstico de zonas criticas } \\
\text { Número de muestras analizadas } \\
\text { por sitio }\end{array}$ \\
\hline & $\begin{array}{l}\text { Creación de un } \\
\text { subgrupo de } \\
\text { expertos en el } \\
\text { área sanitaria }\end{array}$ & $\begin{array}{l}\text { SAG Universidades } \\
\text { CONAF }\end{array}$ & Reunión de expertos & $\begin{array}{l}\text { Constitución del Comité de } \\
\text { expertos }\end{array}$ \\
\hline & $\begin{array}{l}\text { Creación de un } \\
\text { protocolo de } \\
\text { acción en caso } \\
\text { de muestras } \\
\text { positivas }\end{array}$ & Comité de Expertos & $\begin{array}{l}\text { Reunión de expertos para } \\
\text { generar plan de acción }\end{array}$ & Muestras positivas tratadas \\
\hline
\end{tabular}

